\title{
Absorption, tissue distribution and excretion of pelargonidin and its metabolites following oral administration to rats
}

\author{
Manal Abd El Mohsen ${ }^{1,2}$, Joanne Marks ${ }^{3}$, Gunter Kuhnle², Kevin Moore ${ }^{4}$, Edward Debnam ${ }^{3}$, S. Kaila Srai ${ }^{3}$, \\ Catherine Rice-Evans ${ }^{2}$ and Jeremy P. E. Spencer ${ }^{1} *$ \\ ${ }^{1}$ Molecular Nutrition Group, School of Food Biosciences, University of Reading, PO Box 226, Whiteknights, Reading RG6 6AP, UK \\ ${ }^{2}$ Antioxidant Research Group, Wolfson Centre for Age-Related Diseases, GKT School of Biomedical Sciences, King's College, \\ London SE1 9RT, UK \\ ${ }^{3}$ Department of Physiology and Department of Biochemistry \& Molecular Biology, Royal Free and University College Medical \\ School, Royal Free Campus, London NW3 2PF, UK \\ ${ }^{4}$ Centre of Hepatology, Department of Medicine, Royal Free and University College Medical School, Royal Free Campus, \\ London NW3 2PF, UK
}

(Received 21 April 2005 - Revised 23 June 2005 - Accepted 4 August 2005)

\begin{abstract}
Recent reports have demonstrated various cardiovascular and neurological benefits associated with the consumption of foods rich in anthocyanidins. However, information regarding absorption, metabolism, and especially, tissue distribution are only beginning to accumulate. In the present study, we investigated the occurrence and the kinetics of various circulating pelargonidin metabolites, and we aimed at providing initial information with regard to tissue distribution. Based on HPLC and LC-MS analyses we demonstrate that pelargonidin is absorbed and present in plasma following oral gavage to rats. In addition, the main structurally related pelargonidin metabolite identified in plasma and urine was pelargonidin glucuronide. Furthermore, $p$-hydroxybenzoic acid, a ring fission product of pelargonidin, was detected in plasma and urine samples obtained at 2 and $18 \mathrm{~h}$ after ingestion. At $2 \mathrm{~h}$ post-gavage, pelargonidin glucuronide was the major metabolite detected in kidney and liver, with levels reaching 0.5 and $0.15 \mathrm{nmol}$ pelargonidin equivalents/g tissue, respectively. Brain and lung tissues contained detectable levels of the aglycone, with the glucuronide also present in the lungs. Other tissues, including spleen and heart, did not contain detectable levels of pelargonidin or ensuing metabolites. At $18 \mathrm{~h}$ post-gavage, tissue analyses did not reveal detectable levels of the aglycone nor of pelargonidin glucuronides. Taken together, our results demonstrate that the overall uptake of the administered pelargonidin was $18 \%$ after $2 \mathrm{~h}$, with the majority of the detected levels located in the stomach. However, the amounts recovered dropped to $1.2 \%$ only $18 \mathrm{~h}$ post-gavage, with the urine and faecal content constituting almost $90 \%$ of the total recovered pelargonidin.
\end{abstract}

Anthocyanidin: Pelargonidin: Absorption: Distribution: Metabolism

As part of the flavonoid family, anthocyanins are polyphenolic compounds that are widely distributed among pigmented fruit and vegetables. Anthocyanidins are particularly abundant in berries, such as blueberry and blackcurrant, as well as in red wine, thus their presence in the diet can be significant. As potential components of the human diet, previous research on anthocyanidins has concentrated on their biological activities, and included the assessment of possible health benefits. For example, previous dietary interventions in man and animals have demonstrated that the consumption of anthocyanin-rich red wine (Renaud \& de Lorgeril, 1992) or blueberry extract (Joseph et al. 1999) may exert beneficial effects by lowering the risk of $\mathrm{CHD}$, and by retarding age-related declines in neurological function, respectively.

To evaluate the health benefits of anthocyanidins in man it is important to understand their absorption, metabolism, tissue distribution and excretion. Several studies have shown that anthocyanins are absorbed as glycosides that could be recovered from plasma and urine after oral administration (Cao \& Prior, 1999; Miyazawa et al. 1999). However, the bioavailability of anthocyanins is very limited and their metabolism is still not fully understood. In addition, only a few studies (Tsuda et al. 1999; Matsumoto et al. 2001) have quantitatively evaluated the absorption of anthocyanins using purified compounds (directly administered using a defined dose) as opposed to feeding enriched foods or food extracts. To date, there are few data available with regard to anthocyanidin distribution among various body tissues where they could exert their biological effects through antioxidant potential, by association with the cell membrane or through direct intracellular mechanisms. Anthocyanidins are known to be stable under acidic conditions but rapidly broken down under neutral conditions (Brouillard, 1988) such as those encountered in vivo. It is thus necessary to characterise and to quantify anthocyanin metabolites, including ring-fission products, as well as the ingested form of anthocyanidins. In the present study, we 
investigated metabolism and distribution of the strawberry anthocyanin, pelargonidin, after gavage into rats, and assessed the effect of time on the nature and the circulating levels of its metabolites.

\section{Experimental methods}

\section{Animals}

Male Sprague-Dawley rats $(250 \mathrm{~g})$ were obtained from the Comparative Biology Unit at the Royal Free and University College Medical School, and maintained on standard rat chow (Diet RM1; SDS Ltd, Witham, UK). Two groups of rats $(n$ 6) were administered pelargonidin (Extrasynthese, purity $99.82 \%$ ), dissolved in $50 \%$ aqueous ethanol, in a dose of $50 \mathrm{mg} / \mathrm{kg}$ body weight and killed 2 or $18 \mathrm{~h}$ postgavage. Three animals served as control group and received only $50 \%$ aqueous ethanol. In all groups, animals were fasted for $4 \mathrm{~h}$ before pelargonidin administration.

At the selected times, animals were anaesthetised by intraperitoneal injection of pentobarbitone sodium $(90 \mathrm{mg} / \mathrm{kg}$; Sagital; Rhone-Merieux, Harlow, UK). Heparin (0.3 ml, $5000 \mathrm{IU} / \mathrm{ml}$; CP Pharmaceuticals Ltd, Wrexham, UK) was injected via a tail vein injection to reduce blood clotting followed by collection of $2 \mathrm{ml}$ blood by cardiac puncture into heparinised tubes. Animals were exsanguinated with $200 \mathrm{ml}$ ice-cold heparinised $0.9 \%(\mathrm{w} / \mathrm{v})$ saline and the blood-free organs were dissected out immediately. Plasma samples were separated by centrifugation at $600 \mathrm{~g}$ for $30 \mathrm{~min}$ and immediately acidified with $0.44 \mathrm{M}$-trifluoroacetic acid (TFA) in the ratio 5:1. The $\mathrm{Hb}$ concentrations in the cardiac blood sample and exsanguinated fluid was measured using a $\mathrm{Hb}$ assay kit (Sigma, Poole, UK) to allow calculation of blood volume. The removed tissues, liver, kidneys, spleen, heart, small intestine, large intestine, lung and brain, were weighed and then homogenised in TFA (10 volumes/g tissue) using a Ultra Turrax homogeniser (Janke \& Kunkel, Staufen, Germany) for $40 \mathrm{~s}$. Urine and faeces from rats were collected in metabolic cages containing $1.5 \mathrm{ml}$ TFA to prevent the degradation of pelargonidin. Intestinal contents were drained by finger pressure, flushed with TFA, and contents kept for analysis.

\section{Sample preparation}

Plasma $(150 \mu \mathrm{l})$ was de-proteinised with $30 \mu \mathrm{l} 20 \%$ (w/v) TCA then extracted with $200 \mu \mathrm{l}$ ice-cold methanol. HPLC analysis of re-extracted pellet showed no detectable retained material. Solvents contained in the extract obtained after centrifugation were evaporated under $\mathrm{N}$ and the residue was dissolved in $250 \mu \mathrm{l} 20 \%$ (v/v) methanol in $0.44 \mathrm{M}$-TFA for quantitative HPLC analysis. An additional aliquot of plasma was loaded on to an ODS solid extraction cartridge (Sep-Pak C18; Waters, Milford, MA, USA) after the column was washed with $3 \mathrm{ml}$ methanol and equilibrated with $6 \mathrm{ml}$ watermethanol-acetic acid (94:5:1, by vol.) prior to the application of the sample. Following the sample application, the cartridge was washed with the same equilibrating mixture and the anthocyanins were eluted from the cartridge material with $2 \mathrm{ml}$ methanol. The extract was dried under $\mathrm{N}$, and the residue dissolved in $250 \mu 120 \%(\mathrm{v} / \mathrm{v})$ methanol in $0.44 \mathrm{M}$-TFA.
Urine samples were filtered through a micropore filter (Millex-GP, 0.22 mm filter unit; Millipore, Billerica, MA, USA). Extraction of pelargonidin and its metabolites from rat tissues was performed by mixing the homogenates with an equal volume of ice-cold methanol followed by vigorous vortexing for $1 \mathrm{~min}$. The methanolic extract obtained after centrifugation was dried under vacuum at $30^{\circ} \mathrm{C}$ by rotary evaporation and then the extracts were treated as described earlier.

Quantitative determination of pelargonidin and its metabolites was based on external standards. An internal standard, rutin, was added to the samples before extraction and to the external standards to a final concentration of $5 \mu \mathrm{M}$. Calibration curves were conduced over the range of $0 \cdot 5-20 \mu \mathrm{mol} / \mathrm{l}$. Peak area ratios (compound/internal standard) were plotted against concentration of the compound/metabolite. The concentration of pelargonidin glucuronide was calculated using peak area of standard pelargonidin aglycone and expressed as pelargonidin equivalents as previously used for calculation of anthocyanidin metabolites (Felgines et al. 2002; Wu et al. 2002). Calibration curves obtained were linear over the entire range with correlation coefficient values $\geq 0.995$.

\section{Methods of analysis}

HPLC analysis was undertaken using a Waters system (Milford, MA, USA) consisting of controller 600, auto sampler 717 plus, photodiode array detector 996 and on-line degasser. Samples were analysed on a Zorbax SB-C18 column $(4.6 \times 250 \mathrm{~mm}$, with $5 \mu \mathrm{m}$ particle size and a guard column of the same material, $4.6 \times 15 \mathrm{~mm}$ ). Column temperature was set at $30^{\circ} \mathrm{C}$. Mobile phase A consisted of $5 \%$ formic acid aqueous solution and mobile phase B of pure methanol. The gradient applied was as follows: from 0 to $5 \mathrm{~min} 95 \%$ $\mathrm{A}$ and $5 \% \mathrm{~B}$, from 5 to $40 \mathrm{~min}$ to $100 \% \mathrm{~B}$, from 40 to $45 \min 100 \% \mathrm{~B}$, and from $45.1 \mathrm{~min} 90 \% \mathrm{~A}$ and $10 \% \mathrm{~B}$. Run time was $60 \mathrm{~min}$ followed by a $10 \mathrm{~min}$ delay prior to the next injection. Components were identified according to retention times and UV/visible spectra. Detection of pelargonidin was undertaken at $520 \mathrm{~nm}$, internal standard at $300 \mathrm{~nm}$ and phenolic acids at $260 \mathrm{~nm}$. LC-MS analysis was employed using a Finnigan LCQ Deca XP quadrupole ion trap mass spectrometer (San Jose, CA, USA). Separation was performed using a C18 column $(50 \times 2.5 \mathrm{~mm}$; Phenomenex, Schlieren, Switzerland) with the following gradient (phase A: $0.1 \%$ formic acid in water, phase B: $50 \%$ acetonitrile in water, $0.1 \%$ formic acid). The LC-MS gradient was $0-5 \mathrm{~min}$ $100 \%$ A, 5-40 min from $100 \%$ A to $50 \%$ A, 40-60 min to $0 \% \mathrm{~A}, 60-65 \min 0 \% \mathrm{~A}$. Compounds were detected using a full ion scan and identified by performing product ion scans on selected ions. GC-MS analysis was used as described previously (Rechner et al. 2002) to confirm the identity of phenolic acids detected by HPLC.

\section{Calculations and statistics}

Values are given as means with their standard errors of the mean. Statistical analysis was performed using an unpaired $t$ test (Instat) and considered significant at $P<0.05$. 


\section{Results}

The present study investigated the metabolism of pelargonidin after oral administration to rats. HPLC analysis with photodiode array detection was employed to identify the nature of metabolites (Fig. 1). Whereas no anthocyanins were detected in untreated controls, the oral administration of pelargonidin to rats led to the detection of a circulating
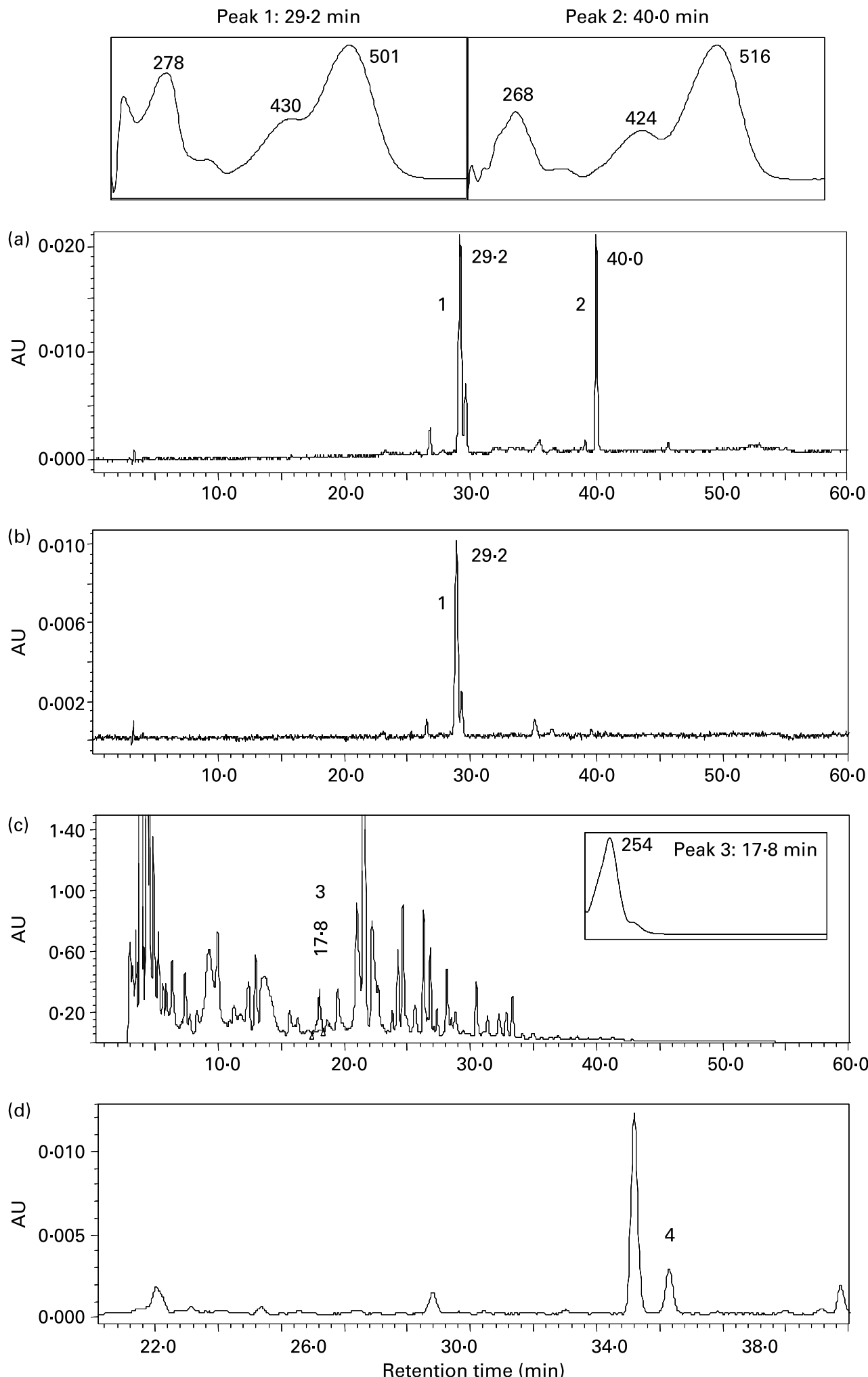

Fig. 1. Representative chromatograms of tissues obtained from pelargonidin-fed rats. (a), Small intestine extract at $520 \mathrm{~nm}$ : peak 1, pelargonidin- $O$ - $\beta$-D-glucuronides; peak 2, pelargonidin. (b), Plasma extract at $520 \mathrm{~nm}$. (c), Urine at $260 \mathrm{~nm}$ : peak 3, p-hydroxybenzoic acid. (d), Plasma at $260 \mathrm{~nm}$ : peak 4, unidentified. AU, absorbance units. 
anthocyanidin at $2 \mathrm{~h}$ post-ingestion. This compound was detected by HPLC, and eluted at a retention time of 40.0 min (peak 2), which corresponds to the retention time of free pelargonidin aglycone. In addition, a major peak at 29.2 min (peak 1), which partially co-eluted with a minor peak, was also detected in the gastrointestinal tract, plasma and urine. Similarities regarding their absorbance spectra as well as the difficulties we experienced in attempting to completely separate these two peaks using various HPLC gradient conditions may indicate that these compounds are structurally related. Both compounds were more hydrophilic than pelargonidin, and thus were predicted to represent glucuronidated
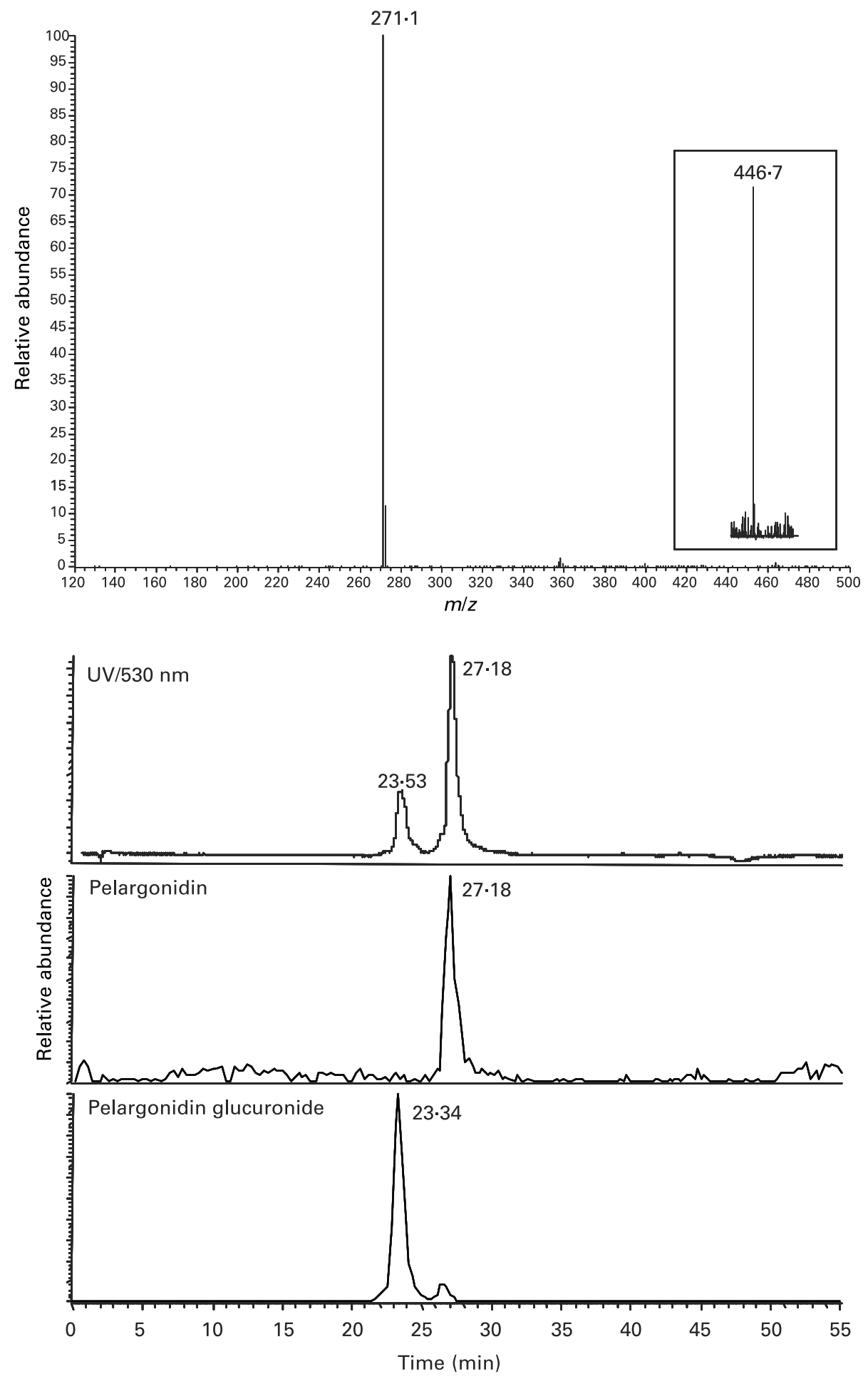

Fig. 2. MS/MS trace of small intestine extract obtained from pelargonidin-gavaged rats. The trace shows pelargonidin $(\mathrm{m} / z=271)$ eluting at $27 \cdot 18 \mathrm{~min}$ and pelargonidin-O-glucuronide $(\mathrm{m} / \mathrm{z}=447)$ with a retention time of $23.34 \mathrm{~min}$. The spectrum shows a single fragment at $\mathrm{m} / \mathrm{z}=271$, the $\mathrm{M}^{+}$ion of the aglycone, indicating the neutral loss of glucuronic acid. The insert shows the spectrum of the base peak $\left(\mathrm{M}^{+}\right)$. 
derivatives of pelargonidin. Thus, a subsequent LC-MS/MS analysis of the isolated peaks was undertaken to confirm the molecular mass for pelargonidin glucuronides (Fig. 2). Two major peaks are detected at $530 \mathrm{~nm}$, one for pelargonidin with $\mathrm{m} / \mathrm{z}=271$ (retention time $27.18 \mathrm{~min}$ ), preceded by a more polar glucuronide signal $\mathrm{m} / \mathrm{z}=447$ (retention time $23.53 \mathrm{~min}$ ) (Fig. 2). The product ion spectrum of the major pelargonidin-glucuronide shows the neutral loss of glucuronic acid, and therefore the major peak is the $\mathrm{M}^{+}$ion of the aglycone.

HPLC chromatograms of urine samples collected for $18 \mathrm{~h}$ post-gavage at $260 \mathrm{~nm}$ (Fig. 1(c)) also revealed the presence of peak 3, eluting at $18 \mathrm{~min}$. This peak was identified as $p$-hydroxybenzoic acid by comparison with an authentic standard compound, and based on retention times and UV spectra as well as by GC-MS analysis following derivatisation (Fig. 3). This phenolic acid was also detected in plasma samples obtained at 2 or $18 \mathrm{~h}$ post-ingestion. Fig. 4 shows two modes of the C-ring fission of pelargonidin, which yield ultimately the detected product, $p$-hydroxybenzoic acid. The dotted line shows breakage of the $\mathrm{C}$-ring between $\mathrm{C} 8 \mathrm{a}-\mathrm{O} 1$ and $\mathrm{C} 2-\mathrm{C} 3$ leading to formation of $p$-hydroxybenzoic acid directly. However, as shown by the dashed line, breakage of the C-ring can also occur between $\mathrm{O} 1-\mathrm{C} 2$ and $\mathrm{C} 4-\mathrm{C} 4 \mathrm{a}$, leading to the formation of $p$-hydroxyphenyl propionic acid, which could be subjected to $\beta$-oxidation leading to formation of $p$-hydroxybenzoic acid, as previously described by Scheline (1991).

In addition, $2 \mathrm{~h}$ plasma samples revealed the presence of an additional peak eluting at $35.2 \mathrm{~min}$ (Fig. 1(d)), which was found to be present at lower levels after $18 \mathrm{~h}$, but which was not detectable in any of the control samples. This finding may be indicative for the presence of another metabolic product resulting from pelargonidin intake. However, we were not able to structurally identify this compound as a pelargonidin metabolite.

Levels of pelargonidin metabolites at 2 and $18 \mathrm{~h}$ were calculated in plasma and tissues and expressed as nmol pelargonidin equivalents/ml plasma or g tissue (Fig. 5). At $2 \mathrm{~h}$ post-gavage, pelargonidin glucuronide was the major metabolite detected in kidney and liver, with levels reaching 0.5 and $0.15 \mathrm{nmol}$ pelargonidin equivalents/g tissue, respectively (Fig. 5(a)). Whereas the brain and the lungs showed the aglycone as the main metabolite with similar levels reaching $0.16 \mathrm{nmol} / \mathrm{g}$, in other tissues, mainly the spleen and heart, no compounds were detected. At $18 \mathrm{~h}$ post-gavage, our tissue analyses did not reveal the presence of any detectable levels of pelargonidin aglycone or its glucuronides. However, the colonic metabolite $p$-hydroxybenzoic acid was present in plasma, reaching $44 \%$ of total metabolites detected at $2 \mathrm{~h}$ postgavage, and being the only metabolite detected in plasma at $18 \mathrm{~h}$ post-gavage (Fig. $5(\mathrm{~b})$ ).

Table 1 shows the levels of metabolites detected in different parts of the gastrointestinal tract at 2 and $18 \mathrm{~h}$ following the administration of pelargonidin to rats. Analyses of the stomach content following the administration of pelargonidin demonstrated the presence of $p$-hydroxybenzoic acid, which accounts for approximately $11 \%$ of the detected compounds. High levels of glucuronides were detected in the small intestine tissue extract $2 \mathrm{~h}$ post-gavage. At $18 \mathrm{~h}$ post-gavage, these metabolites disappeared completely from the small intestine, which would be consistent with the time-dependency of passage through the gastrointestinal tract. However, $p$-hydroxybenzoic acid was detected in the large intestine, both at 2 and $18 \mathrm{~h}$ post-gavage (Table 1$)$.

Analysis of urine samples obtained by aspirating the urine directly from the rat bladder $2 \mathrm{~h}$ post-gavage demonstrated the presence of pelargonidin glucuronide as the only metabolite excreted into the urine at this time. However, $p$-hydroxybenzoic acid constituted approximately $50 \%$ of metabolites found in urine collected for $18 \mathrm{~h}$ post-gavage (Table 2). In contrast to the urine, the glucuronide conjugates were not found in any of the faecal samples, with $p$-hydroxybenzoic acid as the major product. The results reveal that the total recovery of the administered pelargonidin was only $18 \%$

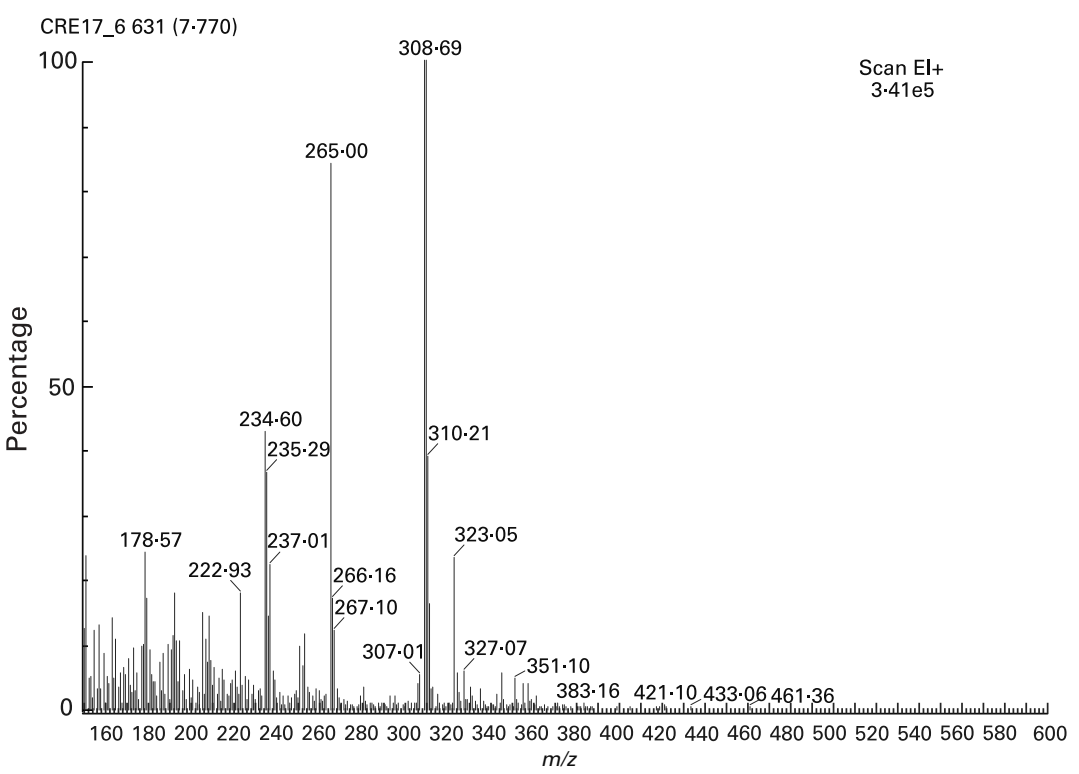

Fig. 3. Mass spectra of the derivatised derivatives of $p$-hydroxybenzoic acid as detected in urine. The spectra shows a characteristic fragment at $m / z=309$, resulting from the loss of a $t$-butyl group of the derivatised compound. The identification was done by comparison with the spectra of the purified standard. 


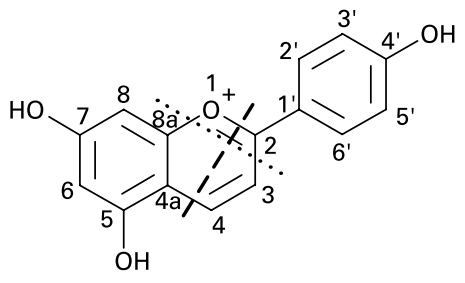

Pelargonidin

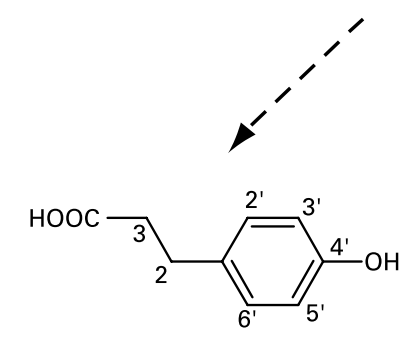

p-Hydroxyphenyl propionic acid

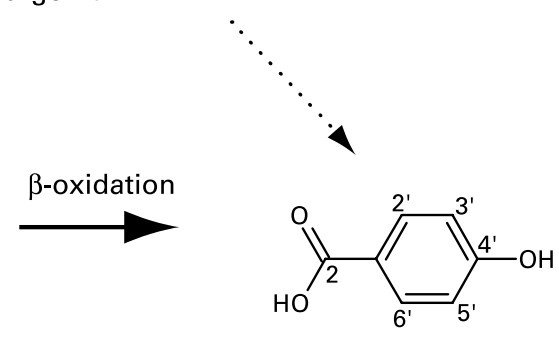

p-Hydroxybenzoic acid

Fig. 4. Possible mechanism for degradation of pelargonidin leading to formation of $p$-hydroxybenzoic acid. ... breakage of C-ring between C8a-O1 and C2-C3 leading to formation of $p$-hydroxybenzoic acid. - - - breakage between O1-C2 and C4-C4a leading to the formation of $p$-hydroxyphenyl propionic acid.

after $2 \mathrm{~h}$, where the majority of the detected levels were located in the stomach; however, the amounts recovered dropped to $1.2 \%$ only $18 \mathrm{~h}$ post-gavage, with the urine and faecal content constituting almost $90 \%$ of the total recovered pelargonidin.
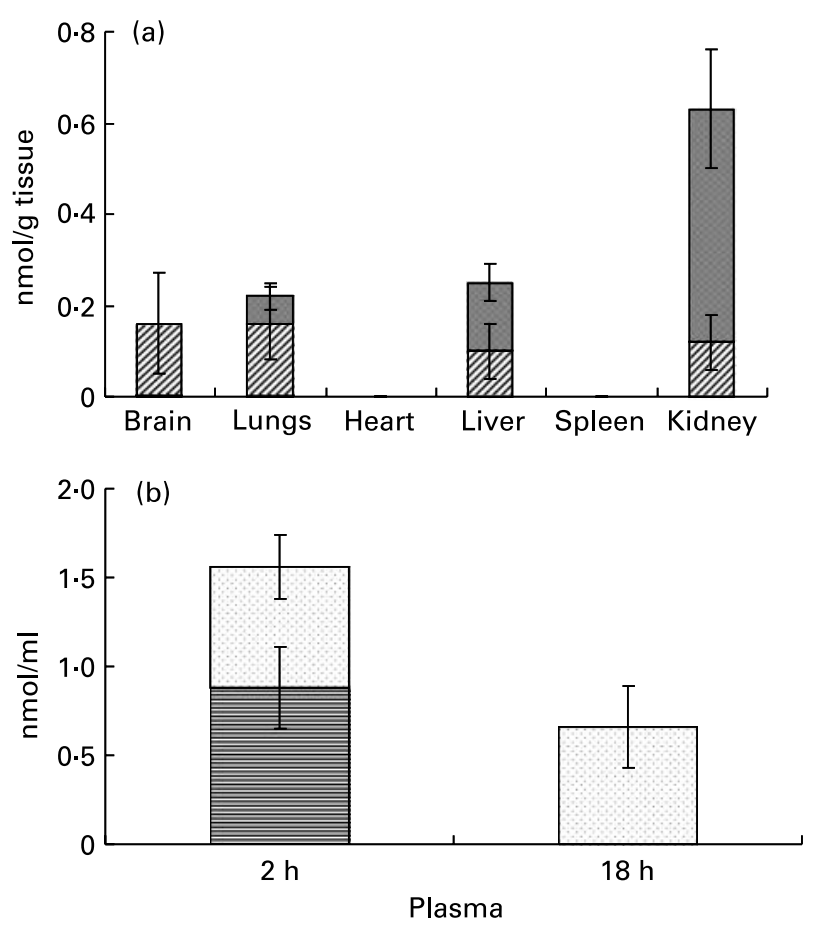

Fig. 5. Concentrations of pelargonidin and its metabolites (calculated as $\mathrm{nmol}$ pelargonidin equivalents) in rat tissues obtained $2 \mathrm{~h}$ (a) and plasma obtained at 2 and $18 \mathrm{~h} \mathrm{(b)} \mathrm{after} \mathrm{oral} \mathrm{administration} \mathrm{of} 50 \mathrm{mg} / \mathrm{kg}$ body weight of pelargonidin. For details of procedures, see p. 52. (a), Pelargonidin in free

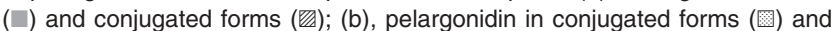
$p$-hydroxybenzoic acid (旦). Values are means with their standard errors depicted by vertical bars (six animals per group).

\section{Discussion}

Recently, much attention has been given to the biological functions of flavonoids. Foods that are rich in anthocyanins have been shown to exert multiple beneficial health effects. In this context, the bioavailability of anthocyanins represents a timely question that we were aiming to address by conducting the present study on the absorption, metabolism, distribution and excretion of pelargonidin.

Our results demonstrated that pelargonidin was absorbed from the gastrointestinal tract and it appeared in the plasma and urine mainly as a glucuronidated conjugate. It has been well documented previously that anthocyanidins are absorbed, in both man and animals, as their intact glycosidic forms present in fruit (Miyazawa et al. 1999; Tsuda et al. 1999). Despite this, various glucuronide conjugates of cyanidin (Wu et al. 2002) as well as pelargonidin (Felgines et al. 2003) have been detected in human plasma previously following the intake of elderberries and strawberries. The mechanism of glucuronide formation from the anthocyanidin glucoside was reported to involve the conversion of the glucoside directly into its corresponding glucuronide (Wu et al. 2002) by the action of UDP-glucose dehydrogenase. Furthermore, anthocyanidin glucosides can be hydrolysed yielding the aglycone, which can be subject to glucuronidation in the intestine and liver. Interestingly, the presence of cyanidin aglycone has been previously reported in the rat jejunum after ingestion of cyanidin-3-glucoside (Tsuda et al. 1999). The ingestion of pelargonidin aglycone and the ensuing detection of circulating pelargonidin glucuronides, in the present study, provides evidence that the flavylium cation structure does not impart resistance to enzymatic conversion by UDP-glucuronosyl transferase, as previously assumed by Miyazawa et al. (1999).

In spite of detecting pelargonidin and its glucuronide in rat plasma and tissues $2 \mathrm{~h}$ after administration, neither the native compound nor its metabolites were found $18 \mathrm{~h}$ after ingestion. A pharmacokinetic study regarding the absorption of anthocyanins has previously demonstrated that these compounds 
Table 1. Total amounts of metabolites (nmol) detected in the gastrointestinal content, 2 and $18 \mathrm{~h}$ following the oral administration of $50 \mathrm{mg} / \mathrm{kg}$ body weight of pelargonidin to rats*

Mean values with their standard errors (six animals per group)

\begin{tabular}{|c|c|c|c|c|c|c|c|c|c|c|c|c|}
\hline & \multicolumn{6}{|c|}{$2 \mathrm{~h}$} & \multicolumn{6}{|c|}{$18 \mathrm{~h}$} \\
\hline & \multicolumn{2}{|c|}{$\begin{array}{l}\text { Pelargonidin } \\
\text { aglycone }\end{array}$} & \multicolumn{2}{|c|}{$\begin{array}{l}\text { Pelargonidin } \\
\text { glucuronide }\end{array}$} & \multicolumn{2}{|c|}{$\mathrm{p}-\mathrm{HBA}$} & \multicolumn{2}{|c|}{$\begin{array}{l}\text { Pelargonidin } \\
\text { aglycone }\end{array}$} & \multicolumn{2}{|c|}{$\begin{array}{l}\text { Pelargonidin } \\
\text { glucuronide }\end{array}$} & \multicolumn{2}{|c|}{$\mathrm{p}-\mathrm{HBA}$} \\
\hline & Mean & SEM & Mean & SEM & Mean & SEM & Mean & SEM & Mean & SEM & Mean & SEM \\
\hline Stomach & 6131 & 995 & ND & ND & 790 & 209 & ND & ND & ND & ND & ND & ND \\
\hline Small intestine & 195 & $62 \cdot 1$ & 189 & $41 \cdot 6$ & 232 & 65.5 & ND & ND & ND & ND & ND & ND \\
\hline Large Intestine & $8 \cdot 7$ & $6 \cdot 1$ & $0 \cdot 25$ & 0.17 & 39.7 & $35 \cdot 1$ & ND & ND & ND & ND & $52 \cdot 5$ & 31.5 \\
\hline
\end{tabular}

ND, non-detectable; p-HBA, $p$-hydroxybenzoic acid.

${ }^{*}$ For details of procedures, see p. 52.

are rapidly absorbed; reaching maximum plasma concentrations of $3.49 \mu \mathrm{M}$ at $15 \mathrm{~min}$ after a single oral dose was administered to rats. Thereafter the plasma concentration fell rapidly reaching $0 \cdot 14 \mu$ Mat $240 \mathrm{~min}$, and thus suggesting a relatively short half-life (Miyazawa et al. 1999). Rapid absorption could be attributed to the involvement of stomach-dependent mechanisms, possibly through a bilitranslocase-mediated mechanism as indicated following administration of grape anthocyanins to rats (Passamonti et al. 2003). The high instability of anthocyanidins at physiological $\mathrm{pH}$ and their rapid decomposition to ring-opened forms and/or degradation products is probably responsible for the relatively short half-life in biological fluids. Indeed, it was recently shown that the flavylium cation form is not regenerated by acidification following incubation of aglycones with control urine or plasma (Felgines et al. 2002).

One of the major findings of the present study was the identification of $p$-hydroxybenzoic acid as an important metabolite of pelargonidin in the rat plasma and gastrointestinal tissues. Despite the fact that this metabolite could also be derived from the metabolism of other compounds such as aromatic amino acids, the high levels detected post-gavage of pelargonidin, as compared with levels detected in control animals, support the notion that this phenolic acid commonly arises from the metabolism of pelargonidin. Indeed, a structurally similar metabolite, namely protocatechuic acid, was detected following an oral cyanidin glucoside administration to rats, with plasma levels reaching eight times that of the anthocyanidin (Tsuda et al. 1999). The detection of $p$-hydroxybenzoic acid in stomach extract and plasma $2 \mathrm{~h}$ after gavage indicates that its formation likely results due to the instability and degradation of the anthocyanidin. However, phenolic acid derivatives may also be formed by the colonic

Table 2. Total amounts of pelargonidin and its metabolites (nmol) excreted in rat urine and faeces collected for $18 \mathrm{~h}$ post-gavage*

Mean values with their standard errors (six animals per group)

\begin{tabular}{lccccc}
\hline & \multicolumn{2}{c}{ Urine } & & \multicolumn{2}{c}{ Faeces } \\
\cline { 2 - 3 } \cline { 6 - 7 } & Mean & SEM & & Mean & SEM \\
\hline Pelargonidin & ND & - & & 182 & 14.7 \\
Pelargonidin glucuronide & 158 & $14 \cdot 8$ & & ND & - \\
$p$-Hydroxybenzoic acid & 155 & 31.3 & & 161 & $15 \cdot 7$ \\
\hline
\end{tabular}

ND, non-detectable.

${ }^{*}$ For details of procedures, see p. 52. microflora. A comparison of the metabolites formed after oral and intraperitoneal administration of catechin, and the suppression of their formation after administration of antibiotics, confirms the catabolising activities of colonic microflora (Das \& Griffiths, 1968). In addition, it was shown recently that in vitro incubation of anthocyanins with human faecal suspension yields phenolic acids as major products (Fleschhut et al. 2005). Our results, demonstrating that a similar pathway exists in vivo, clearly point to the importance of colonic metabolism of flavonoids. Indeed, these metabolites might be equally as important since anticancer properties of aromatic acids have been previously documented (Samid et al. 1997; Thibout et al. 1999).

Considering all pelargonidin metabolites detected, the percentages of products excreted over $18 \mathrm{~h}$ accounted only for $1.2 \%$ of the gavaged dose. Similar findings were also reported where total urinary excretion of blackberry anthocyanidins in rats (Felgines et al. 2002) as well as strawberry anthocyanin metabolites in human subjects (Felgines et al. 2003) corresponded to only 0.93 and $1.8 \%$ of the anthocyanidins ingested, respectively, pointing to the existence of other non-detectable metabolites.

In conclusion, the present results suggest the importance of investigating the biological activities of the degradation products, which could be formed at a later stage, possibly in the colon, when evaluating the properties of diet-derived anthocyanidins.

\section{Acknowledgements}

The authors acknowledge the Food Standards Agency and the Biotechnology and Biological Sciences Research Council (grant no. BB/C518222/1) for financial support on this project.

\section{References}

Brouillard R (1988) The Flavonoids. London: Chapman and Hall. Cao G \& Prior RL (1999) Anthocyanins are detected in human plasma after oral administration of an elderberry extract. Clin Chem 45, 574-576.

Das NP \& Griffiths LA (1968) Studies on flavonoid metabolism. Metabolism of $(+)$-catechin in the guinea pig. Biochem $J \mathbf{1 1 0}$, 449-456.

Felgines C, Talavera S, Gonthier MP, Texier O, Scalbert A, Lamaison JL \& Remesy C (2003) Strawberry anthocyanins are recovered in 
urine as glucuro- and sulfoconjugates in humans. $J$ Nutr $\mathbf{1 3 3}$, $1296-1301$.

Felgines C, Texier O, Besson C, Fraisse D, Lamaison JL \& Remesy C (2002) Blackberry anthocyanins are slightly bioavailable in rats. $J$ Nutr 132, 1249-1253.

Fleschhut J, Kratzer F, Rechkemmer G \& Kulling SE (2005) Stability and biotransformation of anthocyanins in vitro. Eur J Nutr $\mathbf{1 8}$ (Epublication ahead of print version).

Joseph JA, Shukitt-Hale B, Denisova NA, Bielinski D, Martin A, McEwen JJ \& Bickford PC (1999) Reversals of age-related declines in neuronal signal transduction, cognitive, and motor behavioral deficits with blueberry, spinach, or strawberry dietary supplementation. J Neurosci 19, 8114-8121.

Matsumoto H, Inaba H, Kishi M, Tominaga S, Hirayama M \& Tsuda T (2001) Orally administered delphinidin 3-rutinoside and cyanidin 3-rutinoside are directly absorbed in rats and humans and appear in the blood as the intact forms. J Agric Food Chem 49, 1546-1551.

Miyazawa T, Nakagawa K, Kudo M, Muraishi K \& Someya K (1999) Direct intestinal absorption of red fruit anthocyanins, cyanidin3-glucoside and cyanidin-3,5-diglucoside, into rats and humans. J Agric Food Chem 47, 1083-1091.

Passamonti S, Vrhovsek U, Vanzo A \& Mattivi F(2003) The stomach as a site for anthocyanins absorption from food. FEBS Lett 544, 210-213.
Rechner AR, Kuhnle G, Bremner P, Hubbard GP, Moore KP \& Rice-Evans CA (2002) The metabolic fate of dietary polyphenols in humans. Free Radic Biol Med 33, 220-235.

Renaud S \& de Lorgeril M (1992) Wine, alcohol, platelets, and the French paradox for coronary heart disease [comment]. Lancet 339, $1523-1526$.

Samid D, Hudgins WR, Shack S, Liu L, Prasanna P \& Myers CE (1997) Phenylacetate and phenylbutyrate as novel, nontoxic differentiation inducers. Adv Exp Med Biol 400A 501-505.

Scheline RR (1991) Metabolism of oxygen heterocyclic compounds. In Handbook of Mammalian Metabolism of Plant Compounds, pp. 267-290 [RR Scheline, editor]. Boca Raton, FL: CRC Press.

Thibout D, Kraemer M, Di Benedetto M, Saffar L, Gattegno L, Derbin C \& Crepin M (1999) Sodium phenylacetate (NaPa) induces modifications of the proliferation, the adhesion and the cell cycle of tumoral epithelial breast cells. Anticancer Res 19, 2121-2126.

Tsuda T, Horio F \& Osawa T (1999) Absorption and metabolism of cyanidin 3-O-beta-D-glucoside in rats. FEBS Lett $\mathbf{4 4 9}$, $179-182$.

Wu X, Cao G \& Prior RL (2002) Absorption and metabolism of anthocyanins in elderly women after consumption of elderberry or blueberry. J Nutr 132, 1865-1871. 